\title{
Effects of Ramelteon and Other Sleep-Promoting Drugs on Serum Low-Density Lipoprotein and Non-high-density Lipoprotein Cholesterol: A Retrospective Comparative Pilot Study
}

\author{
Haruki Torii, ${ }^{a}$ Rumiko Shimizu, ${ }^{a}$ Yuriko Tanizaki, ${ }^{a}$ Yurina Omiya, ${ }^{a}$ Miwa Yamamoto, ${ }^{a}$ \\ Sayaka Kamiike, ${ }^{a}$ Daisuke Yasuda, ${ }^{a}$ Yoshinori Hiraoka, ${ }^{a}$ Tohru Hashida, ${ }^{b}$ and Noriaki Kume*,a \\ ${ }^{a}$ Division of Clinical Pharmacy, Faculty of Pharmaceutical Sciences, Kobe Gakuin University; 1-1-3 Minatojima, \\ Chuo-ku, Kobe 650-8586, Japan: and ${ }^{b}$ Department of Pharmacy, Kobe City Medical Center General Hospital; 2-1-1 \\ Minatojimaminami-machi, Chuo-ku, Kobe 650-0047, Japan. \\ Received June 1, 2018; accepted September 20, 2018
}

\begin{abstract}
Melatonin has been suggested to play important roles in lipid metabolism as well as circadian rhythm; however, very few studies explored the effects of ramelteon, a selective melatonin receptor agonist, on serum lipid profiles. In this study effects of ramelteon on serum lipid profiles were explored, comparing to those of other sleep-promoting drugs including benzodiazepines and non-benzodiazepines, in patients with insomnia. We retrospectively reviewed medical charts of outpatients who were treated with ramelteon $(8 \mathrm{mg} / \mathrm{d})$ or other sleep-promoting drugs for no less than 8 weeks during the period between October 1st, 2011 and September 30th, 2014, and compared the changes in serum lipid profiles between the two groups. Patients with regular dialysis or malignant diseases treated with cytotoxic anti-cancer drugs, or whose lipid-lowering drugs were altered during the study period, were excluded. Among 365 or 855 outpatients treated with ramelteon or other sleep-promoting drugs, 35 or 46 patients, respectively, had complete serum low-density lipoprotein cholesterol (LDL-C) or non-high-density lipoprotein cholesterol (non-HDL-C) data. Serum LDL-C was significantly reduced from $103.1 \pm 4.4$ to $94.6 \pm 4.2 \mathrm{mg} / \mathrm{dL}(8.2 \%$ reduction, $p<0.05, n=31)$ in the ramelteon group, and was not significantly changed $(p=0.23, n=40)$ in the other sleep-promoting drug group. Non-HDL-C was significantly decreased from $138.8 \pm 6.0$ to $130.6 \pm 4.9 \mathrm{mg} / \mathrm{dL}(5.9 \%$ reduction, $p<0.05, n=32)$ in the ramelteon group, and was not significantly altered $(p=0.29, n=42)$ in the other sleep-promoting drug group. Ramelteon, but not other sleep-promoting drugs, specifically lowers serum LDL-C and non-HDL-C levels.
\end{abstract}

Key words ramelteon; melatonin; insomnia; circadian rhythm; dyslipidemia; lipoprotein

Modern society is referred to as 24 -h society because of night work, shift work and light exposure during the nighttime with the development of civilization. Ohayon and others have reported that prevalence of insomnia in major cities in industrialized countries appeared to be extremely high, and keeps increasing in developed countries. ${ }^{1-4)}$ In Japan, it was nearly $20 \%{ }^{5)}$ In addition to sedentary life styles, shortage in the sleep duration and irregularity in the sleeping time have been shown to provoke and to worsen dyslipidemia in humans. ${ }^{6,7)}$ Patients with insomnia often have risk factors for coronary heart disease (CHD) and atherosclerotic cardiovascular disease (ASCVD), such as diabetes mellitus (DM), dyslipidemia, and history of CHD and ASCVD. ${ }^{8)}$

Heart disease is the second leading cause of death after cancer in Japan ${ }^{9)}$ and often caused by CHD, which is the most prevalent cause of death in the world. ${ }^{10)}$ Hypercholesterolemia, particularly elevated levels of low-density lipoprotein cholesterol (LDL-C), is a major risk factor for CHD and ASCVD. ${ }^{11,12)}$ In addition to LDL-C, non-high-density lipoprotein cholesterol (non-HDL-C) is another important risk factor for CHD and ASCVD in cases with hypertriglyceridemia. ${ }^{13-16)}$ High levels of non-HDL-C, which consists of cholesterol contained in LDL, intermediate-density lipoprotein (IDL), very low-density lipoprotein (VLDL) and chylomicron (CM) remnant, have been demonstrated to be a more potent risk factor for CHD than LDL-C, in cases of hypertriglyceridemia, which is often accompanied by DM. ${ }^{16)}$ Therefore, management of serum LDL-C and non-HDL-C appears to be important for reducing the CHD and ASCVD risk, ${ }^{17-20)}$ and target serum LDL-C and non-HDL-C levels have been assigned according to the risk category for CHD and ASCVD in the 2012 Japan Atherosclerosis Society (JAS) guidelines, ${ }^{21)}$ as well as in the European Atherosclerosis Society (EAS)/European Society of Cardiology (ESC) joint guidelines. ${ }^{22)}$

On the other hand, insomnia and short sleep duration are associated with CHD and ASCVD risk. ${ }^{23)}$ In fact, it has been revealed that the relationship between CHD morbidity and the sleeping duration showed a U-shaped curve in women. ${ }^{24)}$ Melatonin, a hormone secreted from the pineal body mainly during the night time, binds to the melatonin receptor type 1 (MT1 receptor) and the type 2 (MT2 receptor), expressed in the suprachiasmatic nucleus, which controls the circadian rhythm, and induces somnolence. ${ }^{25)}$ Ramelteon has been developed to bind selectively and specifically to these two receptors with high affinity, resulting in the induction of sleep. ${ }^{26,27)}$ A few previous reports showed that melatonin had beneficial effects on the components of metabolic syndrome. ${ }^{28-30)}$ In addition, ramelteon, a melatonin receptor agonist, improved obesity and serum lipid profiles in patients with schizophrenia without $\mathrm{DM}^{31)}$ In the present study, therefore, we have explored the effects of ramelteon on serum LDL-C and nonHDL-C levels in general patients with insomnia by a retrospective medical chart survey. 


\section{PATIENTS AND METHODS}

Study Population and Design Medical charts of the patients, who were treated with ramelteon $(8 \mathrm{mg} / \mathrm{d})$, or other sleep-promoting drugs for, at least, 8 weeks, in the Kobe City Medical Center General Hospital from October 1st, 2011 to September 30th, 2014, were retrospectively reviewed. Patients, whose serum LDL-C or non-HDL-C levels before and after ramelteon or other sleep-promoting drug treatment were available, were enrolled to this study. Patients with regular dialysis for chronic renal failure or malignant diseases treated with cytotoxic anti-cancer drugs, or those who were under seriously ill conditions or whose lipid-lowering medication was altered during the drug treatment, were excluded from this study.

Collection of Patient Medical Data Age, gender, serum lipid, aspartate aminotransferase (AST), alanine transaminase (ALT), $\gamma$-glutamyl transferase $(\gamma$-GTP), creatinine (Cre), uric acid (UA), and glucose (Glc), and plasma glycosylated hemoglobin (HbAlc) levels, accompanying illness and other medication were recorded. Ramelteon treatment for 4-8 weeks, 9-16 weeks and 17-24 weeks were designated as early phase, middle phase, and late phase, respectively. Lipid data at all the three phases were recorded for the each patient. If the lipid levels were measured more than once for the each phase, values at the longest time point from the initiation of the drugs were utilized. LDL-C values were calculated by Frie- dewald's formula if serum triglyceride (TG) levels were less than $400 \mathrm{mg} / \mathrm{dL}$. If serum $\mathrm{TG}$ levels were above $400 \mathrm{mg} / \mathrm{dL}$, direct measurement of LDL-C was utilized. Non-HDL-C levels were also calculated in all the available cases. As controls, serum lipid values were compared between before and after treatment with other sleep-promoting drugs than ramelteon. As additional controls, pre-before LDL-C and non-HDL-C levels (2-24 weeks prior to the initiation of sleep-promoting drugs), which were measured when ramelteon or other sleeppromoting drug treatment had not yet been started, were also evaluated.

Statistical Analysis Continuous values are presented as mean \pm standard error of mean (S.E.M.). Statistical significance in the differences in mean values was analyzed by paired $t$ test or unpaired $t$-test for comparison of paired or unpaired samples, respectively, if the samples were distributed normally and had equal variance. If the samples had non-normal distribution, Mann-Whitney $U$-test was applied. Categorical variables were statistically assessed by Chi-square test or Fisher's exact test. $P$ values less than 0.05 were considered to be statistically significant.

Ethical Approval The protocol of this retrospective study has been approved by ethical committees for human studies in both Kobe City Medical Center General Hospital, and conducted in accordance with the provision of the Declaration of Helsinki (as revised in Tokyo in 2004).

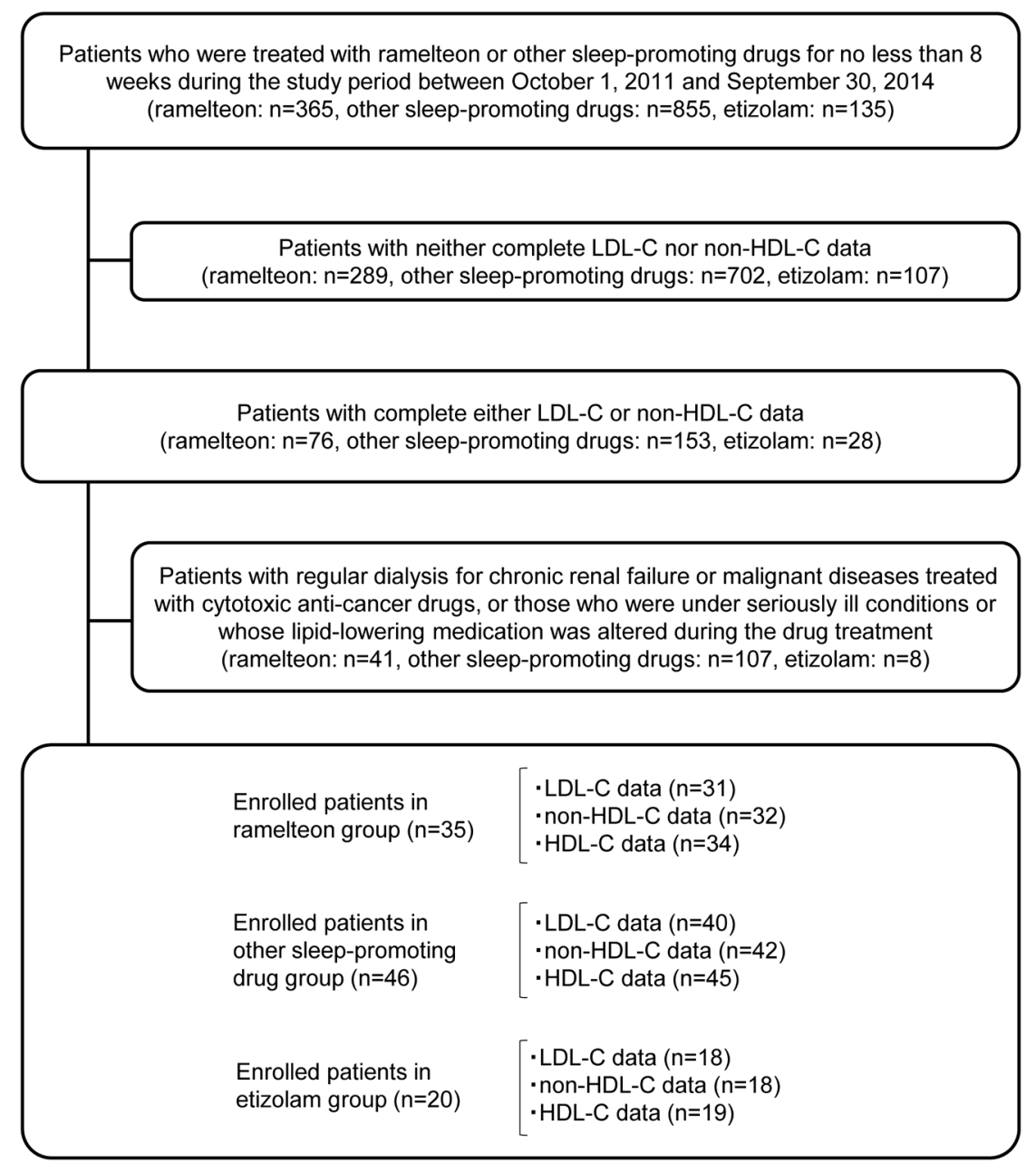

Fig. 1. Schematic Illustration of the Patient Exclusion and Enrollment 


\section{RESULTS}

Patient Characteristics A flow chart of the patient enrollment in this study is shown in Fig. 1. Among 365 or 855 patients who were treated with ramelteon or other sleeppromoting drugs, respectively, for no less than 8 weeks during the study period between October 1st, 2011 and September 30th, 2014, 76 or 153 patients had either complete LDL-C or non-HDL-C data in ramelteon or other sleep-promoting drug groups, respectively. Out of the 76 or 153 patients, 41 or 107 patients were excluded because of regular dialysis for chronic renal failure, malignant diseases treated with cytotoxic anticancer drugs, seriously ill conditions, or alteration of lipidlowering medication during the drug treatment. As a result, 35 (18 males and 17 females) or 46 (20 males and 26 females) patients who had either LDL-C or non-HDL-C data in both before and after ramelteon or other sleep-promoting drug treatment, respectively, were enrolled in this study.

The drug lists and patient numbers of these other sleeppromoting drugs are shown in Table 1. Etizolam and zopiclone were most prevalently prescribed in these patients. Among the patients treated with etizolam, which was the most prevalently

Table 1. Contents of Other Sleep-Promoting Drugs

\begin{tabular}{llc}
\hline \hline Classification & Details & $n=46$ \\
\hline Benzodiazepines & Lormetazepam & 1 \\
& Brotizolam & 6 \\
& Etizolam & 20 \\
Non-benzodiazepines & Zolpidem & 3 \\
& Eszopiclone & 4 \\
& Zopiclone & 12 \\
\hline
\end{tabular}

prescribed drug in the other sleep-promoting drug group, serum LDL-C or non-HDL-C levels were measured both before and after the drug treatment in 20 (10 males and 10 females) patients (Fig. 1).

Characteristics of the patients enrolled in this study are summarized in Table 2. Neither age $(p=0.76, p=0.31)$ nor gender $(p=0.48, p=1.00)$ was significantly different between the ramelteon and other sleep-promoting drug groups or between the ramelteon and etizolam groups. Prevalence of elderly (aged no less than 75 years) patients either was not significantly different. Baseline laboratory data are shown in Table 3. Mean LDL-C, non-HDL-C and HDL-C levels were 103.1, 138.8 and $49.5 \mathrm{mg} / \mathrm{dL}$, respectively, in the ramelteon group, and these values were $109.2,133.2$ and $54.6 \mathrm{mg} / \mathrm{dL}$, respectively, in the other sleep-promoting drug group, and 116.4, 140.3 and $52.6 \mathrm{mg} / \mathrm{dL}$, respectively, in the etizolam group. These differences were not statistically significant in LDL-C $(p=0.33, p=0.08)$ or non-HDL-C $(p=0.74, p=0.80)$. Baseline HDL-C levels were significantly lower in the ramelteon group than in the other sleep-promoting drug group $(p<0.05)$; however, they were not significantly different from those in the etizolam group $(p=0.40)$.

Mean HbA1c values were 6.7, 6.2 and $6.3 \%$ in the ramelteon, other sleep-promoting drug and etizolam groups, respectively, $(p=0.17, p=0.47)$. Mean values of other laboratory data were within the normal limits, and were not significantly different between the ramelteon and other sleep-promoting drug groups or between the ramelteon and etizolam groups. Table 4 shows comorbidities of this study population. Comorbidities were not significantly different, except that heart failure $(p<0.05)$ was significantly less prevalent in the ramelteon group than in the other sleep-promoting drug group but not

Table 2. Characteristics of Enrolled Patients in Ramelteon, Other Sleep-Promoting Drug and Etizolam Groups

\begin{tabular}{|c|c|c|c|c|c|}
\hline & Ramelteon & Other drugs ${ }^{a)}$ & $p$ Value & Etizolam & $p$ Value \\
\hline Total patients (male/female) & $35(18 / 17)$ & $46(20 / 26)$ & $0.48^{b)}$ & $20(10 / 10)$ & $1.00^{b)}$ \\
\hline Elderly (male/female) & $11(4 / 7)$ & $22(7 / 15)$ & $1.00^{c)}$ & $8(2 / 6)$ & $1.00^{c)}$ \\
\hline Non-elderly (male/female) & $24(14 / 10)$ & $24(13 / 11)$ & $0.77^{b)}$ & $12(8 / 4)$ & $0.72^{b)}$ \\
\hline Age (years), mean \pm S.E.M. (range) & $67.1 \pm 2.3(31-86)$ & $67.3 \pm 2.3(28-91)$ & $0.76^{d)}$ & $61.9 \pm 3.8(28-81)$ & $0.31^{d)}$ \\
\hline
\end{tabular}

Values are expressed as n (male/female) or median (range). a) Patients who were prescribed with other sleep-promoting drug, benzodiazepines or non-benzodiazepines, as indicated in Table 1. b) $p$ Value calculated using Chi-square test. c) $p$ Value calculated using Fisher's exact test. $d$ ) $p$ value calculated using Mann-Whitney $U$-test. S.E.M.: standard error of the mean.

Table 3. Baseline Laboratory Data in Ramelteon and Other Sleep-Promoting Drug and Etizolam Groups

\begin{tabular}{|c|c|c|c|c|c|c|c|c|}
\hline \multirow{2}{*}{$\begin{array}{l}\text { Parameters } \\
\text { LDL-C (mg/dL) }\end{array}$} & \multicolumn{2}{|c|}{ Ramelteon } & \multicolumn{2}{|c|}{ Other drugs $^{a}$ ) } & \multirow{2}{*}{$\frac{p \text { Value }}{0.33^{b)}}$} & \multicolumn{2}{|c|}{ Etizolam } & \multirow{2}{*}{$\frac{p \text { Value }}{0.08^{c)}}$} \\
\hline & $103.1 \pm 4.4$ & (31) & $109.2 \pm 4.3$ & $(40)$ & & $116.4 \pm 6.4$ & (18) & \\
\hline Non-HDL-C (mg/dL) & $138.8 \pm 6.0$ & (32) & $133.2 \pm 4.8$ & $(42)$ & $0.74^{c)}$ & $140.3 \pm 7.6$ & $(18)$ & $0.80^{c)}$ \\
\hline HDL-C (mg/dL) & $49.5 \pm 2.7$ & (34) & $54.6 \pm 2.0$ & $(45)$ & $0.04^{c)}$ & $52.6 \pm 3.5$ & (19) & $0.40^{c)}$ \\
\hline $\operatorname{AST}(\mathrm{IU} / \mathrm{L})$ & $22.9 \pm 1.9$ & (34) & $27.4 \pm 2.8$ & (45) & $0.22^{c)}$ & $25.3 \pm 6.0$ & (19) & $0.27^{c)}$ \\
\hline ALT (IU/L) & $22.5 \pm 2.6$ & (34) & $25.9 \pm 3.8$ & (45) & $0.93^{c)}$ & $27.2 \pm 7.3$ & (19) & $0.64^{c)}$ \\
\hline$\gamma$-GTP (IU/L) & $44.6 \pm 10.7$ & $(32)$ & $45.3 \pm 10.1$ & (43) & $0.79^{c)}$ & $49.2 \pm 21.0$ & (18) & $0.63^{c)}$ \\
\hline Cre (mg/dL) & $0.8 \pm 0.05$ & (35) & $1.1 \pm 0.1$ & $(45)$ & $0.30^{c)}$ & $1.0 \pm 0.3$ & (19) & $0.52^{c)}$ \\
\hline UA (mg/dL) & $5.4 \pm 0.2$ & (34) & $5.4 \pm 0.3$ & (43) & $0.92^{b)}$ & $5.3 \pm 0.4$ & (18) & $0.78^{b)}$ \\
\hline Glc (mg/dL) & $136.7 \pm 9.3$ & (21) & $127.1 \pm 9.4$ & $(35)$ & $0.15^{c)}$ & $130.9 \pm 18.5$ & (14) & $0.30^{c)}$ \\
\hline HbA1c (\%) & $6.7 \pm 0.3$ & (22) & $6.2 \pm 0.2$ & (24) & $0.17^{c)}$ & $6.3 \pm 0.3$ & (11) & $0.47^{c)}$ \\
\hline
\end{tabular}

Values are expressed as mean \pm S.E.M. $(n)$. a) Patients who were prescribed with other sleep-promoting drugs, benzodiazepines or non-benzodiazepines, as indicated in Table 1 . b) $p$ Value calculated using Unpaired $t$-test. c) $p$ Value calculated using Mann-Whitney $U$-test. S.E.M.: standard error of the mean, LDL-C: low-density lipoprotein cholesterol, Non-HDL-C: non-high-density lipoprotein cholesterol, HDL-C: high-density lipoprotein cholesterol, AST: aspartate aminotransferase, ALT: alanine transaminase, $\gamma$-GTP: $\gamma$-glutamyltransferase, Cre: creatinine, UA: uric acid, Glc: glucose, HbA1c: glycated hemoglobin A1c. 
than in the etizolam group. Interestingly, DM, hypertension, dyslipidemia and hyperuricemia were shared by $68.6,48.6$, 48.6 and $22.9 \%$, respectively, in the ramelteon group, by 47.8 , $56.5,43.5$ and $19.6 \%$, respectively, in the other sleep-promoting drug group, and by 50.0, 35.0, 20.0, and 5.0\%, respectively, in the etizolam group. Thus, these study population, who were subscribed ramelteon or other sleep-promoting medication, including etizolam, showed high and similar prevalence of certain types of metabolic disorder. Moreover, CHD was shared by $28.6,28.3$ and $15.0 \%$ of the patients in the ramelteon, other sleep-promoting drug and etizolam groups, respectively, which were not significantly different.

Table 5 shows concomitant medication in this study population. Prevalence of concomitant drugs was not significantly different between the two groups, except that anti-diabetic drugs $(p<0.05)$ and dipeptidyl peptidase-4 (DPP-4) inhibitors $(p<0.05)$ were significantly more prevalently prescribed in the ramelteon group (54.3 and $34.3 \%$, respectively) when compared to the other sleep-promoting drug group (28.3 and $13.0 \%$, respectively), but not when compared to the etizolam group (40.0 and $25.0 \%$, respectively). Moreover, lipid-lowering drugs, anti-hypertensive drugs and anti-thrombotic drugs were subscribed in $45.7,71.4$ and $54.3 \%$ of the patients, respectively, in the ramelteon group. These rates were 52.2, 65.2 and $56.5 \%$, respectively, in the other sleep-promoting drug group, and were $45.0,55.0$ and $55.0 \%$, respectively, in the etizolam group. These data were not significantly different, and appeared to reflect the patient characteristics shown in Table 4.

In combination with ramelteon, additional benzodiazepines, non-benzodiazepines or barbiturates were administered in $7(20.0 \%), 9(25.7 \%)$ or $1(2.9 \%)$ patients, respectively. In patients treated with other sleep-promoting medication, additional benzodiazepines, non-benzodiazepines or barbiturates were prescribed in $13(28.3 \%), 9(19.6 \%)$ or $1(2.2 \%)$ patients. These differences were statistically insignificant $(p=0.39$, $p=0.51$ and $p=1.00$, respectively)

Effects of Ramelteon, Other Sleep-Promoting Drugs and Etizolam on LDL-C, Non-HDL-C and HDL-C Levels Serum LDL-C, non-HDL-C, and HDL-C levels were compared between before and after the administration of ramelteon $(8 \mathrm{mg} / \mathrm{d})$ or other sleep-promoting medication (Fig. 2). LDL-C levels were significantly reduced from $103.1 \pm 4.4$ to $94.6 \pm 4.2 \mathrm{mg} / \mathrm{dL}(8.2 \%$ reduction, $p<0.05, n=31)$ in the ramelteon group. In contrast, they were not significantly changed

Table 4. Comorbidities of Ramelteon, Other Sleep-Promoting Drug and Etizolam Groups

\begin{tabular}{|c|c|c|c|c|c|c|}
\hline Classification & Detail & Ramelteon & Other drugs ${ }^{a)}$ & $p$ Value & Etizolam & $p$ Value \\
\hline \multirow[t]{2}{*}{ Cancer } & Solid cancer & $5.7 \quad(2)$ & $19.6 \quad(9)$ & $0.10^{c)}$ & 20.0 & $0.18^{c)}$ \\
\hline & Hematologic cancer & $2.9 \quad(1)$ & $(0)$ & $0.43^{c)}$ & $0.0 \quad(0)$ & $1.00^{c)}$ \\
\hline \multirow[t]{3}{*}{ Heart disease } & Coronary heart disease & $28.6(10)$ & $28.3(13)$ & $0.98^{b)}$ & 15.0 & $0.33^{c)}$ \\
\hline & Heart failure & $11.4 \quad(4)$ & $32.6(15)$ & $0.03^{b)}$ & $5.0 \quad(1)$ & $0.64^{c)}$ \\
\hline & Arrhythmia & $20.0 \quad(7)$ & 39.1 & $0.06^{b)}$ & $10.0 \quad(2)$ & $0.46^{c)}$ \\
\hline \multirow[t]{2}{*}{ Cerebrovascular disease } & Non-cardiogenic cerebral infarction & $14.3 \quad(5)$ & $10.9 \quad(5)$ & $0.74^{c)}$ & $0.0 \quad(0)$ & $0.15^{c)}$ \\
\hline & Cerebral hemorrhage & $5.7 \quad(2)$ & $2.2 \quad(1)$ & $0.58^{c)}$ & $0.0 \quad(0)$ & $0.53^{c)}$ \\
\hline Peripheral arterial disease & Arteriosclerosis obliterans & $2.9 \quad(1)$ & $15.2 \quad(7)$ & $0.13^{c)}$ & $5.0 \quad(1)$ & $1.00^{c)}$ \\
\hline \multirow[t]{8}{*}{ Endocrine metabolic disease } & Diabetes mellitus & $68.6(24)$ & 47.8 (22) & $0.06^{b)}$ & 50.0 & $0.17^{b)}$ \\
\hline & Hypertension & $48.6 \quad(17)$ & $56.5(26)$ & $0.48^{b)}$ & $35.0 \quad(7)$ & $0.33^{b)}$ \\
\hline & Dyslipidemia & $48.6 \quad(17)$ & $43.5(20)$ & $0.65^{b)}$ & $20.0 \quad$ (4) & $0.80^{b)}$ \\
\hline & Hyperuricemia & $22.9 \quad(8)$ & $19.6 \quad(9)$ & $0.72^{b)}$ & 5.0 & $0.13^{c)}$ \\
\hline & Hyperthyroidism & $0.0 \quad(0)$ & $6.5 \quad(3)$ & $0.25^{c)}$ & 0.0 & $1.00^{c)}$ \\
\hline & Hypothyroidism & $2.9 \quad(1)$ & $8.7 \quad$ (4) & $0.38^{c)}$ & 0.0 & $1.00^{c)}$ \\
\hline & Pheochromocytoma & $0.0 \quad(0)$ & $2.2 \quad(1)$ & $1.00^{c)}$ & 0.0 & $1.00^{c)}$ \\
\hline & Osteoporosis & $2.9 \quad(1)$ & $6.5 \quad(3)$ & $0.63^{c)}$ & $0.0 \quad(0)$ & $1.00^{c)}$ \\
\hline \multirow[t]{3}{*}{ Liver disease } & Fatty liver & 8.6 & 4.3 & $0.65^{c)}$ & 0.0 & $0.29^{c)}$ \\
\hline & Hepatitis B virus & $5.7 \quad(2)$ & $0.0 \quad(0)$ & $0.18^{c)}$ & 0.0 & $0.53^{c)}$ \\
\hline & Hepatitis $\mathrm{C}$ virus & $5.7 \quad(2)$ & $0.0 \quad(0)$ & $0.18^{c)}$ & 0.0 & $0.53^{c)}$ \\
\hline Kidney disease & Chronic kidney disease & 34.3 (12) & $43.5(20)$ & $0.57^{b)}$ & 20.0 & $0.19^{b)}$ \\
\hline \multirow[t]{4}{*}{ Respiratory disease } & Bronchial asthma & $8.6 \quad(3)$ & $8.7 \quad$ (4) & $1.00^{c)}$ & 5.0 & $1.00^{c)}$ \\
\hline & Mycobacterium tuberculosis & 2.9 & (1) & $1.00^{c)}$ & 0.0 & $1.00^{c)}$ \\
\hline & Chronic Obstructive Pulmonary Disease & 2.9 & (1) & $1.00^{c)}$ & 0.0 & $1.00^{c)}$ \\
\hline & Interstitial pneumonia & 2.9 & $6.5 \quad(3)$ & $0.63^{c)}$ & $0.0 \quad(0)$ & $1.00^{c)}$ \\
\hline \multirow[t]{4}{*}{ Central nervous system disease } & Epilepsy & 5.7 & 8.7 & $0.69^{c)}$ & 10.0 & $0.62^{c)}$ \\
\hline & Clinical depression & 2.9 & 15.2 & $0.29^{c)}$ & 15.0 & $0.34^{c)}$ \\
\hline & Schizophrenia & 0.0 & 6.5 & $0.25^{c)}$ & 0.0 & $1.00^{c)}$ \\
\hline & Alzheimer's disease & 0.0 & (1) & $1.00^{c)}$ & 0.0 & $1.00^{c)}$ \\
\hline \multirow[t]{2}{*}{ Autoimmune disease } & Rheumatoid arthritis & 0.0 & 6.5 & $0.25^{c)}$ & 0.0 & $1.00^{c)}$ \\
\hline & Systemic lupus erythematosus & 0.0 & 2.2 & $1.00^{c)}$ & 0.0 & $1.00^{c)}$ \\
\hline
\end{tabular}

Values are expressed as percentages $(n) . a$ ) Patients who were prescribed with other sleep-promoting drugs, benzodiazepines or non-benzodiazepines, as indicated in Table 1 . b) $p$ Value calculated using Chi-square test. c) $p$ Value calculated using Fisher's exact test. 
Table 5. Concomitant Medication in Ramelteon, Other Sleep-Promoting Drug and Etizolam Groups

\begin{tabular}{|c|c|c|c|c|c|c|c|c|c|c|}
\hline Classification & Ramelteon & $\begin{array}{l}\text { Other } \\
\text { drugs }^{a)}\end{array}$ & $\begin{array}{c}p \\
\text { Value }\end{array}$ & Etizolam & $\begin{array}{c}p \\
\text { Value }\end{array}$ & Ramelteon & $\begin{array}{l}\text { Other } \\
\left.\text { drugs }^{a}\right)\end{array}$ & $\begin{array}{c}p \\
\text { Value }\end{array}$ & Etizolam & $\begin{array}{c}p \\
\text { Value }\end{array}$ \\
\hline \multirow[t]{4}{*}{ Lipid-lowering drugs } & $45.7(16)$ & $52.2(24)$ & $0.56^{b)}$ & $45.0 \quad(9)$ & $0.96^{b)} \mathrm{HMG}-\mathrm{CoA}$ reductase inhibitors & $42.9(15)$ & $43.5(20)$ & $0.96^{b)}$ & $45.0(9)$ & $0.88^{b)}$ \\
\hline & & & & & NPC1L1 inhibitor ezetimibe & $8.6 \quad(3)$ & $4.3 \quad(2)$ & $0.65^{c}$ & $5.0(1)$ & $1.00^{c)}$ \\
\hline & & & & & Omega-3 fatty acids & $5.7(2)$ & 8.7 (4) & $0.69^{c}$ & $5.0(1)$ & $1.00^{c)}$ \\
\hline & & & & & Nicotinic acid derivative & $0.0 \quad(0)$ & $4.3(2)$ & $0.50^{c)}$ & $0.0(0)$ & $1.00^{c)}$ \\
\hline \multirow[t]{7}{*}{ Anti-diabetic drugs } & $54.3(19)$ & 28.3 & $0.02^{b)}$ & $40.0 \quad(8)$ & $0.31^{b)}$ DPP-4 inhibitors & $34.3(12)$ & $13.0 \quad(6)$ & $0.02^{b)}$ & $25.0(5)$ & $0.47^{b)}$ \\
\hline & & & & & Biguanides & $17.1 \quad(6)$ & $8.7 \quad(4)$ & $0.32^{c}$ & $10.0(2)$ & $0.70^{c}$ \\
\hline & & & & & Sulfonylureas & $8.6 \quad(3)$ & $13.0 \quad(6)$ & $0.72^{c}$ & $20.0(4)$ & $0.24^{c}$ \\
\hline & & & & & $\alpha$-Glucosidase inhibitors & $5.7 \quad(2)$ & 2.2 (1) & $0.58^{c}$ & $5.0(1)$ & $1.00^{c)}$ \\
\hline & & & & & Aldose reductase inhibitors & $2.9(1)$ & $0.0(0)$ & $0.43^{c}$ & $0.0(0)$ & $1.00^{c}$ \\
\hline & & & & & Thiazolidine derivative & $2.9(1)$ & $2.2(1)$ & $1.00^{c}$ & $5.0(1)$ & $1.00^{c)}$ \\
\hline & & & & & Rapid-acting insulin secretagogue & $0.0(0)$ & $4.3(2)$ & $0.50^{c}$ & $5.0(1)$ & $0.36^{c)}$ \\
\hline \multirow[t]{7}{*}{ Anti-hypertensive drugs } & $71.4(25)$ & $65.2(30)$ & $0.55^{b)}$ & 55.0 & $0.22^{b)}$ Calcium channel blockers & $45.7(16)$ & $34.8(16)$ & $0.32^{b)}$ & $40.0(8)$ & $0.68^{b)}$ \\
\hline & & & & & Angiotensin II receptor blockers & $40.0(14)$ & $32.6(15)$ & $0.49^{b)}$ & $25.0(5)$ & $0.26^{b)}$ \\
\hline & & & & & Beta adrenergic blocking drugs & $40.0(14)$ & $32.6(15)$ & $0.49^{b)}$ & $35.0(7)$ & $0.71^{b)}$ \\
\hline & & & & & Loop diuretics & 20.0 & $32.6(15)$ & $0.21^{b)}$ & $10.0(2)$ & $0.46^{b)}$ \\
\hline & & & & & Aldosterone receptor antagonists & 11.4 (4) & $8.7 \quad(4)$ & $0.72^{c)}$ & $5.0(1)$ & $0.64^{c)}$ \\
\hline & & & & & Thiazide diuretics & 2.9 & $6.5(3)$ & $0.63^{c)}$ & $0.0(0)$ & $1.00^{c}$ \\
\hline & & & & & Vasopressin receptor antagonists & $0.0(0)$ & $2.2(1)$ & $1.00^{c)}$ & $0.0(0)$ & $1.00^{c)}$ \\
\hline \multirow[t]{2}{*}{ Anti-hyperuricemic drugs } & $20.0 \quad(7)$ & 21.7 & $0.85^{b)}$ & 15.0 & $0.73^{c}$ Urate synthesis inhibitors & $17.1 \quad(6)$ & $21.7(10)$ & $0.61^{b)}$ & $15.0(3)$ & $1.00^{c)}$ \\
\hline & & & & & Uricosuric drugs & $2.9 \quad(1)$ & $0.0 \quad(0)$ & $0.43^{c)}$ & $0.0(0)$ & $1.00^{c)}$ \\
\hline \multirow[t]{2}{*}{ Anti-thrombotic drugs } & $54.3(19)$ & 56.5 & $0.84^{b)}$ & 55.0 & $0.96^{b)}$ Antiplatelet drugs & $45.7(16)$ & $37.0(17)$ & $0.43^{b)}$ & $40.0(8)$ & $0.68^{b)}$ \\
\hline & & & & & Novel oral anticoagulants & $8.6 \quad(3)$ & $0.0 \quad(0)$ & $0.08^{c)}$ & $0.0(0)$ & $0.29^{c)}$ \\
\hline \multirow[t]{3}{*}{ Other heart disease drugs } & $25.7 \quad(9)$ & $21.7(10)$ & $0.68^{b)}$ & 15.0 & $0.50^{c}$ Organic nitrates & $8.6(3)$ & $6.5(3)$ & $1.00^{c)}$ & $10.0(2)$ & $1.00^{c)}$ \\
\hline & & & & & Antiarrhythmic drugs & 11.4 & $13.0 \quad(6)$ & $1.00^{c)}$ & $5.0(1)$ & $0.64^{c)}$ \\
\hline & & & & & Drugs for heart failure & $8.6 \quad(3)$ & $10.9 \quad(5)$ & $1.00^{c)}$ & $25.0(5)$ & $0.12^{c)}$ \\
\hline \multirow{3}{*}{$\begin{array}{l}\text { Sleep-inducing drugs/ } \\
\text { anti-anxiety drugs }\end{array}$} & $40.0(14)$ & $39.1(18)$ & $0.94^{b)}$ & $60.0(12)$ & $0.15^{b)}$ Benzodiazepines & $20.0 \quad(7)$ & $28.3(13)$ & $0.39^{b)}$ & $35.0(7)$ & $0.22^{b)}$ \\
\hline & & & & & Non-benzodiazepines & 25.7 (9) & $19.6(9)$ & $0.51^{b)}$ & $35.0(7)$ & $0.47^{b)}$ \\
\hline & & & & & Barbiturate & $2.9 \quad(1)$ & 2.2 & $1.00^{c)}$ & $5.0(1)$ & $1.00^{c)}$ \\
\hline \multirow[t]{5}{*}{ Hormone preparations } & $8.6 \quad(3)$ & 10.9 & $1.00^{c)}$ & 15.0 & $0.66^{c)}$ Steroids & $2.9(1)$ & 2.2 & $1.00^{c)}$ & $5.0(1)$ & $1.00^{c)}$ \\
\hline & & & & & Thyroid hormone preparations & $8.6(3)$ & $10.9 \quad(5)$ & $1.00^{c}$ & $15.0(3)$ & $0.66^{c)}$ \\
\hline & & & & & Estrogen preparations & $2.9(1)$ & $0.0 \quad(0)$ & $0.44^{c}$ & $0.0(0)$ & $1.00^{c}$ \\
\hline & & & & & Progesterone preparations & $2.9 \quad(1)$ & $0.0(0)$ & $0.44^{c}$ & $0.0(0)$ & $1.00^{c}$ \\
\hline & & & & & Growth hormone preparations & $2.9(1)$ & $0.0(0)$ & $0.44^{c}$ & $0.0(0)$ & $1.00^{c)}$ \\
\hline \multirow[t]{2}{*}{ Vasodilators } & $0.0 \quad(0)$ & $4.3(2)$ & $0.50^{c}$ & $0.0 \quad(0)$ & $1.00^{c)}$ Prostaglandin & $0.0 \quad(0)$ & $4.3(2)$ & $0.50^{c}$ & $0.0(0)$ & $1.00^{c)}$ \\
\hline & & & & & Endothelin receptor antagonists & $0.0 \quad(0)$ & $2.2(1)$ & $1.00^{c)}$ & $0.0(0)$ & $1.00^{c)}$ \\
\hline \multirow[t]{2}{*}{ Anti-osteoporosis drugs } & $5.7 \quad(2)$ & $8.7 \quad(4)$ & $0.69^{c)}$ & 10.0 & $0.62^{c)}$ Bisphosphonates & $5.7 \quad(2)$ & $2.2(1)$ & $0.58^{c}$ & $5.0(1)$ & $1.00^{c)}$ \\
\hline & & & & & Activated forms of vitamin D3 & $2.9(1)$ & $6.5(3)$ & $0.63^{c)}$ & $5.0(1)$ & $1.00^{c)}$ \\
\hline \multirow[t]{3}{*}{ Typical antipsychotics } & 5.7 (2) & 0.0 & $0.18^{c}$ & $0.0 \quad(0)$ & $0.53^{c}$ Benzamide antipsychotics & $2.9(1)$ & 0.0 & $0.43^{c}$ & $0.0(0)$ & $1.00^{c}$ \\
\hline & & & & & Phenothiazine antipsychotics & 2.9 (1) & 0.0 & $0.43^{c)}$ & $0.0(0)$ & $1.00^{c)}$ \\
\hline & & & & & Butyrophenone antipsychotics & $2.9(1)$ & $0.0(0)$ & $0.43^{c)}$ & $0.0(0)$ & $1.00^{c)}$ \\
\hline \multirow[t]{2}{*}{ Atypical antipsychotics } & $8.6 \quad(3)$ & $6.5(3)$ & $1.00^{c}$ & 5.0 & $1.00^{c)}$ Serotonin-dopamine antagonists & $5.7(2)$ & $6.5(3)$ & $1.00^{c)}$ & $5.0(1)$ & $1.00^{c)}$ \\
\hline & & & & & Dopamine and 5-HT stabilizer quetiapine & 2.9 & $0.0(0)$ & $0.43^{c)}$ & $0.0(0)$ & $1.00^{c)}$ \\
\hline \multirow{4}{*}{$\begin{array}{l}\text { Antidepressants /mood } \\
\text { stabilizer }\end{array}$} & $17.1 \quad(6)$ & 17.4 & $0.98^{c)}$ & $30.0 \quad(6)$ & $0.32^{c)}$ Selective serotonin reuptake inhibitors & $11.4 \quad(4)$ & $10.9 \quad(5)$ & $1.00^{c)}$ & $20.0(4)$ & $0.44^{c)}$ \\
\hline & & & & & Lithium carbonate & $2.9(1)$ & $2.2(1)$ & $1.00^{c}$ & $0.0(0)$ & $1.00^{c)}$ \\
\hline & & & & & Tricyclic antidepressants & $0.0 \quad(0)$ & $2.2(1)$ & $1.00^{c)}$ & $0.0(0)$ & $1.00^{c)}$ \\
\hline & & & & & Sodium valproate & $2.9(1)$ & $8.7 \quad(4)$ & $0.38^{c)}$ & $15.0(3)$ & $0.13^{c)}$ \\
\hline Immunosuppressant drugs & $0.0 \quad(0)$ & $2.2(1)$ & $1.00^{c}$ & $0.0 \quad(0)$ & $1.00^{c)}$ Cyclosporin & $0.0 \quad(0)$ & $2.2(1)$ & $1.00^{c)}$ & $0.0(0)$ & $1.00^{c)}$ \\
\hline \multirow[t]{2}{*}{ Therapeutic drugs for allergy } & $8.6 \quad(3)$ & 4.3 (2) & $0.65^{c}$ & 5.0 & $1.00^{c)}$ Antihistamines & $5.7 \quad(2)$ & $4.3(2)$ & $1.00^{c)}$ & $5.0(1)$ & $1.00^{c)}$ \\
\hline & & & & & Chemical mediator release inhibitors & $2.9(1)$ & $0.0(0)$ & $0.43^{c}$ & $0.0(0)$ & $1.00^{c)}$ \\
\hline Anti-viral drugs & $2.9(1)$ & $0.0(0)$ & $0.43^{c)}$ & $0.0 \quad(0)$ & $1.00^{c)}$ Anti-HBV drugs & $2.9(1)$ & $0.0(0)$ & $0.43^{c}$ & $0.0(0)$ & $1.00^{c}$ \\
\hline
\end{tabular}

Values are expressed as percentages $(n) . a$ ) Patients who were prescribed with other sleep-promoting drugs, benzodiazepines or non-benzodiazepines, as indicated in Table 1. b) $p$ Value calculated using Chi-square test. c) $p$ Value calculated using Fisher's exact test. 
A LDL-C

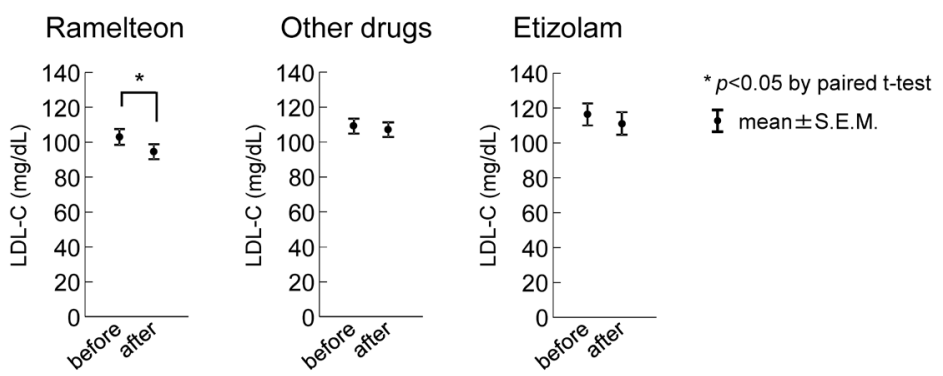

\section{B Non-HDL-C}
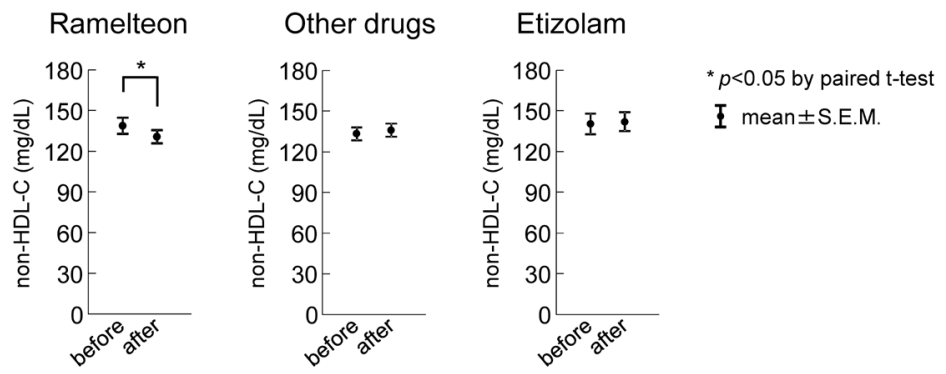

\section{HDL-C}
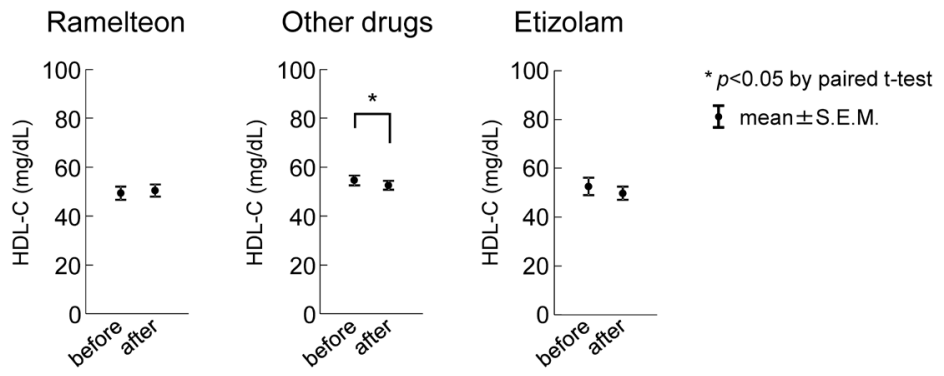

before: values before drug treatment

after: values after drug treatment

Fig. 2. Changes in Serum LDL-C, Non-HDL-C and HDL-C Levels after Treatment with Ramelteon, Other Sleep-Promoting Drugs, or Etizolam in Total Patients

Serum LDL-C (panel A), non-HDL-C (panel B) and HDL-C (panel C) levels before and after treatment with ramelteon or other sleep-promoting drugs, such as benzodiazepines and non-benzodiazepines, are shown. Data are indicated as mean \pm S.E.M.

in the other sleep-promoting drug group (from 109.2 \pm 4.3 to $107.1 \pm 4.2 \mathrm{mg} / \mathrm{dL}, p=0.23, n=40)$ or in the etizolam group (from $116.4 \pm 6.4$ to $111.0 \pm 6.5 \mathrm{mg} / \mathrm{dL}, p=0.14, n=18$ ) (Fig. 2A). Non-HDL-C levels were significantly decreased from $138.8 \pm 6.0$ to $130.6 \pm 4.9 \mathrm{mg} / \mathrm{dL}(5.9 \%$ reduction, $p<0.05, n=32)$ in the ramelteon group, and were not significantly altered in the other sleep-promoting drug group (from 133.2 \pm 4.8 to $135.8 \pm 4.8 \mathrm{mg} / \mathrm{dL}, p=0.29, n=42$ ) or in the etizolam group (from $140.3 \pm 7.6$ to $141.9 \pm 6.9 \mathrm{mg} / \mathrm{dL}, p=0.42, n=18$ ) (Fig. $2 \mathrm{~B}$ ). On the other hand, HDL-C levels were not significantly affected in the ramelteon group $(p=0.26)$, but were significantly decreased in the other sleep-promoting drug group (from $54.6 \pm 2.0$ to $52.6 \pm 1.8 \mathrm{mg} / \mathrm{dL}, 3.5 \%$ reduction, $p<0.05, n=45$ ), and also tended to be decreased in the etizolam group (from $52.6 \pm 3.5$ to $49.8 \pm 2.6 \mathrm{mg} / \mathrm{dL}, 5.3 \%$ reduction, $p=0.06, n=19$ ) although the difference was statistically insignificant.

Because patients with heart failure were significantly less prevalent and baseline serum HDL-C levels were significantly lower in patients treated with ramelteon than those with other sleep-promoting drugs in the present study population, we have explored the possibility whether presence of heart failure or baseline serum HDL-C levels might affect changes in serum LDL-C and non-HDL-C levels. For that purpose, serum LDL-C and non-HDL-C change rates were compared between patients with and without heart failure, as well as between those with baseline HDL-C levels below and above the median value. Serum LDL-C change rates $(\%)$ were $+4.5 \pm 4.0 \%$ and $-5.5 \pm 2.5 \%$ in patients with and without heart failure, respectively $(p=0.05)$. These values for non-HDL-C (median, interquartile ranges) were $-3.6(-9.1,4.3) \%$ and $-1.1(-8.9$, $8.7) \%$, respectively $(p=0.69)$. Serum LDL-C change rates $(\%)$ were $-1.7 \pm 2.7 \%$ and $-4.1 \pm 3.6 \%$ in patients with baseline HDL-C levels above and below the median value, respectively $(p=0.59)$. These values for non-HDL-C (median, interquartile 
A Early phase
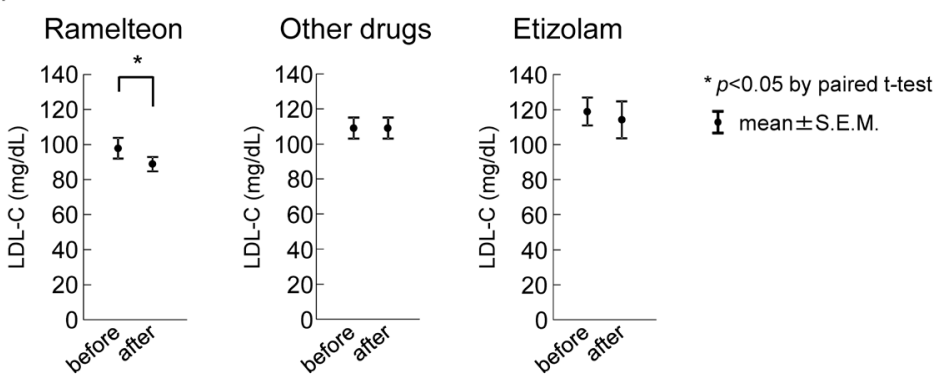

B Middle phase
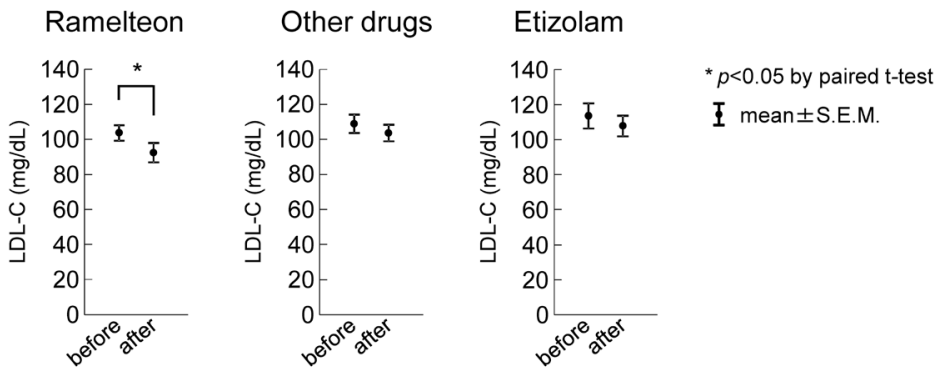

C Late phase
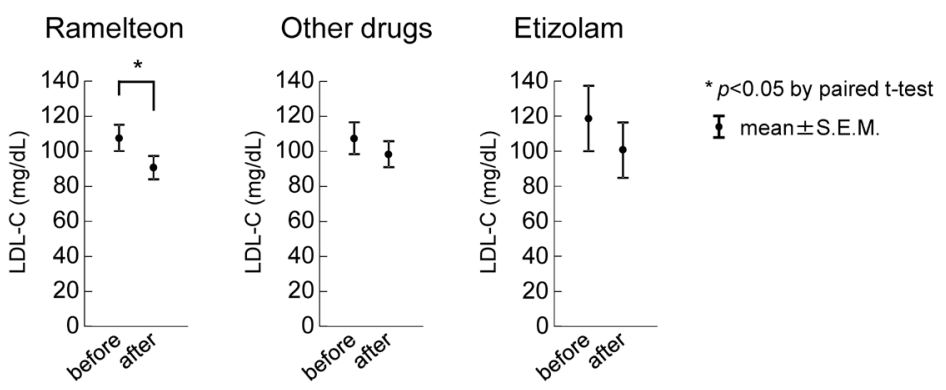

before: values before drug treatment

after: values after drug treatment

Fig. 3. Comparison of Serum LDL-C Changes after Treatment with Ramelteon, Other Sleep-Promoting Drugs or Etizolam among Different Treatment Periods

Serum LDL-C levels before and after treatment with ramelteon, other sleep-promoting drugs or etizolam for 4-8 weeks (early phase, panel A), 9-16 weeks (middle phase, panel B) and 17-24 weeks (late phase, panel C) are shown. Data are indicated as mean \pm S.E.M.

ranges) were $-3.0(-7.5,9.2)$ and $-2.5(-9.6,6.9) \%$, respectively $(p=0.72)$. Thus, neither heart failure nor baseline serum HDL-C levels significantly affected the observed serum LDL-C or non-HDL-C reductions. In addition, prescription rates of DM drugs, as well as DPP-4 inhibitors, were significantly higher in the ramelteon group than in the other sleeppromoting drug group. Therefore, we have further explored whether these drugs might affect serum LDL-C or non-HDL$\mathrm{C}$ changes. Serum LDL-C change rates were $-5.7 \pm 3.7 \%$ and $-1.4 \pm 2.7 \%$ in patients prescribed with and without DM drugs, respectively $(p=0.35, n=71)$. These values for nonHDL-C (median, interquartile ranges) were $-1.2(-8.0,6.3) \%$ and $-3.0(-8.9,10.1) \%$, respectively $(p=0.56, n=74)$. Serum LDL-C change rates were $-4.0 \pm 4.1 \%$ and $-2.8 \pm 2.6 \%$ in patients prescribed with and without DPP-4 inhibitors, respectively $(p=0.82, n=71)$. These values for non-HDL-C (median, interquartile ranges) were $-2.1(-7.8,2.6) \%$ and $-2.9(-8.9$,
$10.1) \%$, respectively $(p=0.38, n=74)$. Thus, neither, DM drugs nor DPP4 inhibitors significantly affected serum LDL-C or non-HDL-C levels.

Furthermore, changes in LDL-C (Fig. 3), non-HDLC (Fig. 4) and HDL-C (Fig. 5) levels between before and after the drug treatment were evaluated separately in the early (after 4-8 weeks), middle (after 9-16 weeks) and late (after 17-24 weeks) phases. In the early phase, LDL-C levels were significantly reduced from 98.0 \pm 5.9 to $88.9 \pm 4.1 \mathrm{mg} / \mathrm{dL}$ in the ramelteon group $(9.3 \%$ reduction, $p<0.05, n=19)$, and were not significantly changed (from $109.0 \pm 6.0$ to $108.9 \pm 6.0 \mathrm{mg} / \mathrm{dL}, p=0.49, n=24$ ) in the other sleep-promoting drug group and (from 118.8 \pm 8.0 to $114.2 \pm 10.6 \mathrm{mg} / \mathrm{dL}, p=0.12, n=9$ ) in the etizolam group (Fig. $3 \mathrm{~A})$. In the middle phase, LDL-C levels were significantly decreased from $103.7 \pm 4.4$ to $92.4 \pm 5.6 \mathrm{mg} / \mathrm{dL}$ ( $10.9 \%$ reduction, $n=20, p<0.05)$ in the ramelteon group, and were not signifi- 
A Early phase

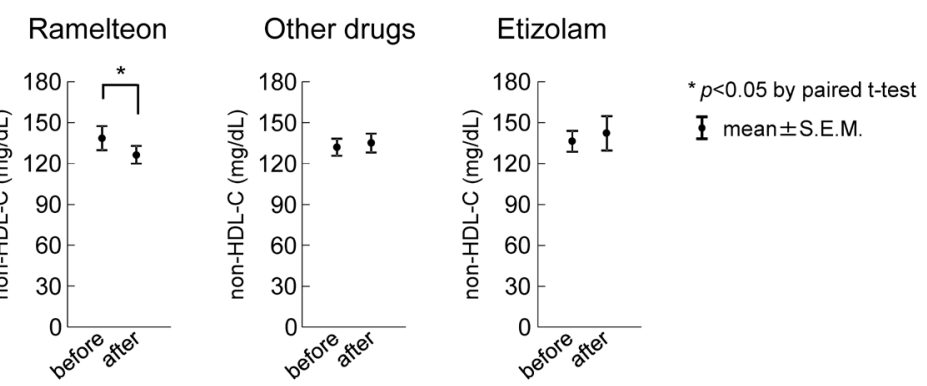

B Middle phase

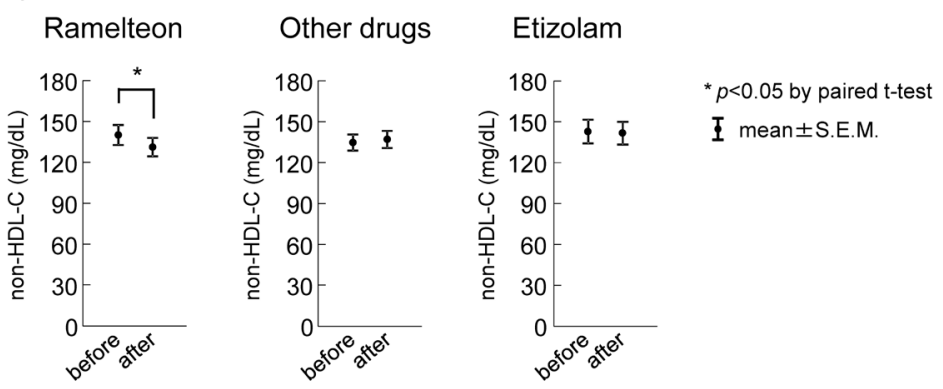

C Late phase

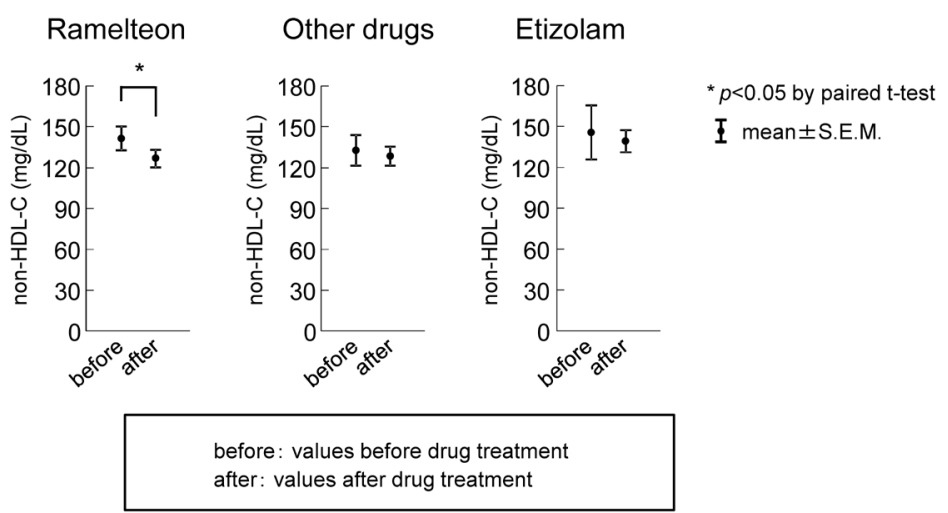

Fig. 4. Comparison of Serum Non-HDL-C Changes after Treatment with Ramelteon, Other Sleep-Promoting Drugs or Etizolam among Different Treatment Periods

Serum non-HDL-C levels before and after treatment with ramelteon, other sleep-promoting drugs or etizolam for 4-8 weeks (early phase, panel A), 9-16 weeks (middle phase, panel B) and 17-24 weeks (late phase, panel C) are shown. Data are indicated as mean \pm S.E.M.

cantly altered (from $109.0 \pm 5.2$ to $103.7 \pm 4.7 \mathrm{mg} / \mathrm{dL}, p=0.13$, $n=22$ ) in the other sleep-promoting drug group and (from $113.5 \pm 7.3$ to $107.8 \pm 5.8 \mathrm{mg} / \mathrm{dL}, p=0.19, n=13)$ in the etizolam group (Fig. 3B). In the late phase, LDL-C levels were significantly declined from $107.6 \pm 7.5$ to $90.8 \pm 6.6 \mathrm{mg} / \mathrm{dL}(15.6 \%$ reduction, $n=15, p<0.05)$ in the ramelteon group, and were not significantly affected (from $107.5 \pm 9.1$ to $98.4 \pm 7.4 \mathrm{mg} / \mathrm{dL}$, $p=0.10, n=12)$ in the other sleep-promoting drug group and (from $118.7 \pm 18.7$ to $100.7 \pm 15.8 \mathrm{mg} / \mathrm{dL}, p=0.14, n=5$ ) in the etizolam group (Fig. 3C). Concerning non-HDL-C levels (Fig. 4 ), in the early phase, non-HDL-C levels were significantly declined from $138.6 \pm 8.8$ to $126.4 \pm 6.6 \mathrm{mg} / \mathrm{dL}(8.8 \%$ reduction, $p<0.05, n=20$ ) in the ramelteon groups, and were not significantly changed (from $131.9 \pm 6.4$ to $135.1 \pm 6.9 \mathrm{mg} / \mathrm{dL}$, $p=0.27, n=25$ ) in the other sleep-promoting drug group and (from $136.4 \pm 7.6$ to $142.3 \pm 12.5 \mathrm{mg} / \mathrm{dL}, p=0.28, n=10$ ) in the etizolam group (Fig. 4A). In the middle phase, non-
HDL-C levels were significantly reduced from 140.0 \pm 7.4 to $131.2 \pm 6.8 \mathrm{mg} / \mathrm{dL} \quad(6.3 \%$ reduction, $p<0.05, n=20)$ in the ramelteon group, and were not significantly altered (from $134.8 \pm 5.9$ to $137.1 \pm 6.3 \mathrm{mg} / \mathrm{dL}, p=0.34, n=26$ ) in the other sleep-promoting drug group and (from 142.9 \pm 8.6 to $141.7 \pm 8.4 \mathrm{mg} / \mathrm{dL}, p=0.43, n=14$ ) in the etizolam group (Fig. $4 \mathrm{~B})$. In the late phase, non-HDL-C levels were significantly declined from $140.9 \pm 8.7$ to $126.1 \pm 6.5 \mathrm{mg} / \mathrm{dL}(10.5 \%$ reduction, $p<0.05, n=16)$ in the ramelteon group, and were not significantly affected (from $132.7 \pm 11.2$ to $128.4 \pm 7.0 \mathrm{mg} / \mathrm{dL}$, $p=0.30, n=13)$ in the other sleep-promoting drug group and (from $145.5 \pm 19.8$ to $139.2 \pm 8.2 \mathrm{mg} / \mathrm{dL}, p=0.36, n=6$ ) in the etizolam group (Fig. 4C). Changes in HDL-C levels were examined separately in the early, middle and late phases in ramelteon and other sleep-promoting drug groups (Fig. 5). In the early phase, HDL-C levels were not significantly altered (from $50.5 \pm 3.6$ to $52.5 \pm 3.7 \mathrm{mg} / \mathrm{dL}, p=0.16, n=22$ ) in 
A Early phase

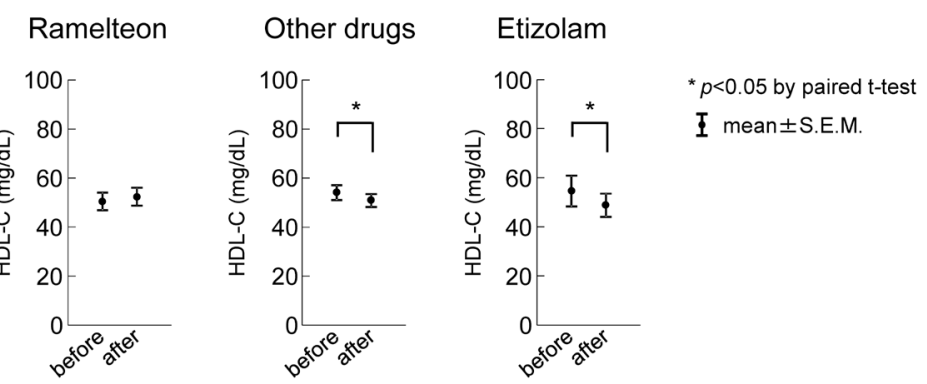

B Middle phase
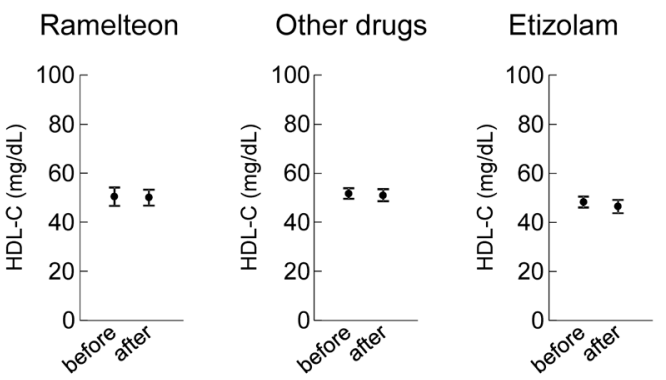

I mean \pm S.E.M.

C Late phase

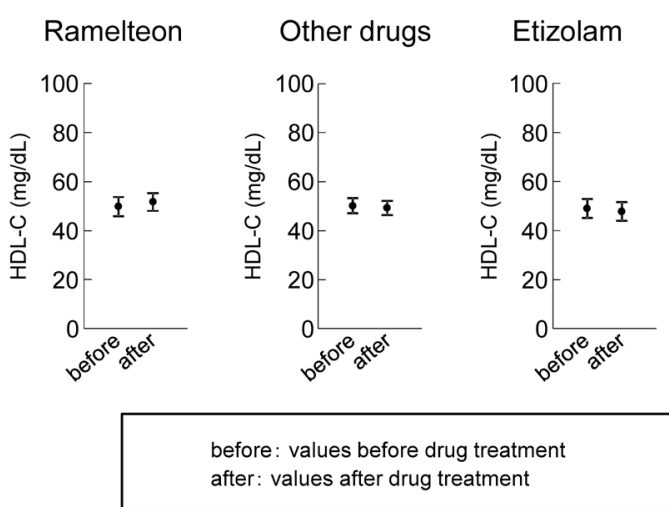

I mean \pm S.E.M.

Fig. 5. Comparison of Serum HDL-C Changes after Treatment with Ramelteon, Other Sleep-Promoting Drugs or Etizolam among Different Treatment Periods

Serum HDL-C levels before and after treatment with ramelteon or other sleep-promoting drugs for 4-8 weeks (early phase, panel A), 9-16 weeks (middle phase, panel B) and 17-24 weeks (late phase, panel C) are shown. Data are indicated as mean \pm S.E.M.

the ramelteon group, but were significantly decreased from $54.1 \pm 3.1$ to $50.9 \pm 2.6 \mathrm{mg} / \mathrm{dL}(5.9 \%$ reduction, $p<0.05, n=26)$ in the other sleep-promoting drug group, additionally, from $54.6 \pm 6.2$ to $48.8 \pm 4.8 \mathrm{mg} / \mathrm{dL}(10.6 \%$ reduction, $p<0.05, n=10)$ in the etizolam group (Fig. 5A). In the middle phase, HDL-C levels were significantly changed neither in the ramelteon group (from $50.4 \pm 3.7$ to $50.0 \pm 3.2 \mathrm{mg} / \mathrm{dL}, p=0.42, n=22$ ), in the other sleep-promoting drug group (from $51.7 \pm 2.1$ to $51.0 \pm 2.5 \mathrm{mg} / \mathrm{dL}, p=0.27, n=27)$ nor in the etizolam group (from $48.2 \pm 2.3$ to $46.4 \pm 2.7 \mathrm{mg} / \mathrm{dL}, p=0.13, n=14$ ) (Fig. $5 \mathrm{~B}$ ). Similarly, in the late phase, HDL-C levels were not significantly altered either in the ramelteon group (from $49.8 \pm 3.9$ to $51.8 \pm 3.6 \mathrm{mg} / \mathrm{dL}, p=0.22, n=18)$, in the other sleep-promoting drug group (from $50.2 \pm 3.1$ to $49.4 \pm 2.9 \mathrm{mg} / \mathrm{dL}, p=0.26, n=14$ ) or in the etizolam group (from $49.0 \pm 3.9$ to $47.9 \pm 3.7 \mathrm{mg} / \mathrm{dL}$, $p=0.33, n=7$ ) (Fig. 5C).

Changes in the LDL-C, Non-HDL-C and HDL-C Levels before Ramelteon Treatment To exclude the possibility if decline in the LDL-C, non-HDL-C and HDL-C levels were natural courses independent of ramelteon or other sleeppromoting drug treatment, changes in the LDL-C, non-HDL$\mathrm{C}$ and HDL-C levels before initiation of ramelteon or other sleep-promoting drugs, were evaluated. Among the 35 or 46 enrolled patients in the ramelteon or the other sleep-promoting drug group, LDL-C, non-HDL-C and HDL-C levels, which were measured at 2-24 weeks prior to the start of ramelteon or other sleep-promoting drug treatment (pre-before values), were available in 24, 25 and 28 patients, or in 18, 20 and 11 patients, respectively. Out of 20 enrolled patients in etizolam group, pre-before values of LDL-C, non-HDL-C and HDL-C levels were available in 6,8 and 8 patients.

Pre-before levels of LDL-C were 98.6 \pm 5.9 or $102.9 \pm 6.9 \mathrm{mg} / \mathrm{dL}$ in patients treated with ramelteon or other sleep-promoting drugs, respectively. Pre-before non-HDL- 


\section{A LDL-C}

\section{Ramelteon}

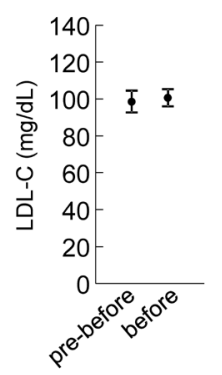

\section{B Non-HDL-C}

\section{Ramelteon}

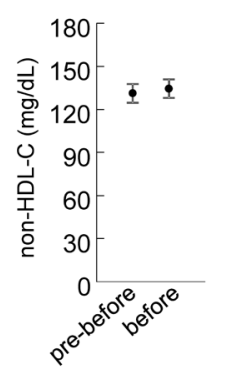

\section{HDL-C}

\section{Ramelteon}

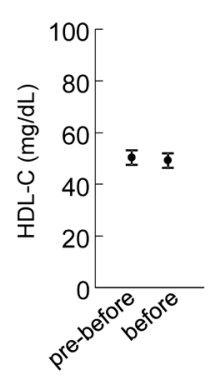

\section{Other drugs}

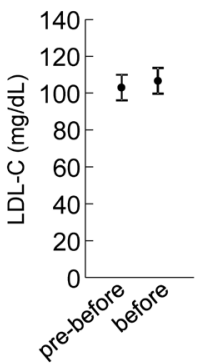

Other drugs

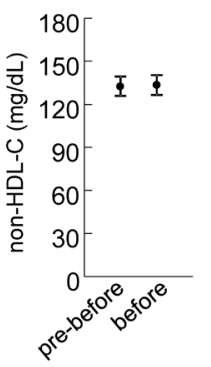

Other drugs

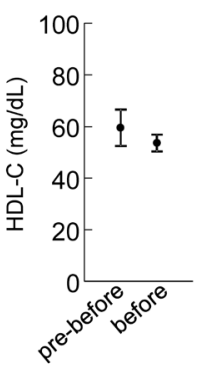

\section{Etizolam}

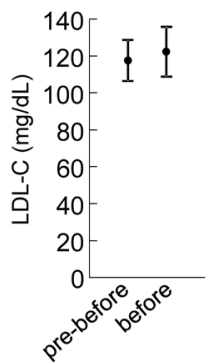

$\Phi$ mean \pm S.E.M.

(1)

\section{Etizolam}

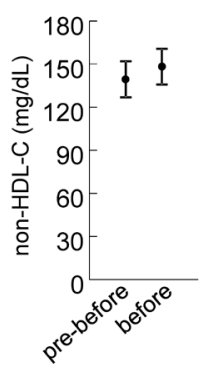

I mean \pm S.E.M.

\section{Etizolam}

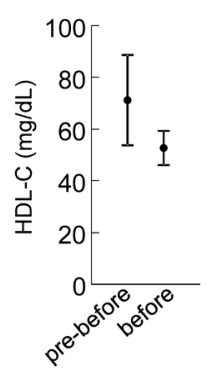

I mean \pm S.E.M.

pre-before: values prior to the initiation of drug treatment

before: values immediately before drug treatment

Fig. 6. Changes in Serum LDL-C, Non-HDL-C and HDL-C Levels Prior to Ramelteon, Other Sleep-Promoting Drug or Etizolam Treatment

LDL-C (panel A), non-HDL-C (panel B) and HDL-C (panel C) levels prior to the initiation of ramelteon, other sleep-promoting drug or etizolam treatment (pre-before levels) and immediately before the treatment with these drugs (before levels) are shown. Data are indicated as mean \pm S.E.M.

C levels were $131.2 \pm 6.6$ or $132.6 \pm 6.7 \mathrm{mg} / \mathrm{dL}$ in patients treated with ramelteon or other sleep-promoting drugs, respectively. Pre-before HDL-C levels were $50.3 \pm 2.8$ or $59.5 \pm 7.1 \mathrm{mg} / \mathrm{dL}$ in patients treated with ramelteon or other sleep-promoting drugs, respectively. Neither LDL-C $(p=0.35$, $p=0.20$ or $p=0.30$, Fig. $6 \mathrm{~A})$, non-HDL-C $(p=0.28, p=0.41$ or $p=0.15$, Fig. $6 \mathrm{~B})$ nor HDL-C $(p=0.47, p=0.21$ or $p=0.17$, Fig. $6 \mathrm{C})$ levels were significantly different between pre-before and before values in patients treated with ramelteon, other sleeppromoting drugs or etizolam, respectively.

\section{DISCUSSION}

In the modern lifestyle, people live under the bright light all the day and night without bright and dark cycles, resulting in less amounts of melatonin secretion. ${ }^{32}$ Sleep disturbance or insomnia keeps increasing in major cities in industrialized countries. ${ }^{1-5)}$ Accordingly, sleep-promoting agents have been more frequently prescribed in recent years, which may make various health problems, including serious side effects and drug addiction. ${ }^{33-35)}$ Ramelteon appears to cause less side effects and drug addiction when compared to other benzodiazepines and non-benzodiazepine sleep-promoting drugs. ${ }^{27)}$

Insomnia and short sleep duration have been shown to be associated with CHD and ASCVD risk. ${ }^{23,24)}$ Elevated levels of serum LDL-C and non-HDL-C are major risk factors for CHD and ASCVD. ${ }^{11-16)}$ To treat insomnia, on the other hand, a variety of sleep-promoting drugs are currently prescribed. A previous study showed that continuous administration of melatonin $(5 \mathrm{mg} / \mathrm{d})$ for 2 months significantly reduced serum 
LDL-C levels, as well as systolic and diastolic blood pressure, compared to the baseline levels in patients with metabolic syndrome. ${ }^{28)}$ Other previous studies also have indicated that melatonin improved components of metabolic syndrome. ${ }^{29)}$ However, another double-blind placebo-controlled crossover randomized trial by melatonin treatment $(8 \mathrm{mg} / \mathrm{d})$ revealed the modest and statistically insignificant decrease in serum LDL-C in metabolic syndrome patients without $\mathrm{DM}^{30}{ }^{30}$ On the other hand, in outpatients with schizophrenia without DM, Borba et al. have shown that ramelteon $(8 \mathrm{mg} / \mathrm{d})$ administration for 8 weeks showed statistically significant decreases in total cholesterol, cholesterol to HDL ratio and LDL particle number, as well as statistically insignificant decline in LDL-C, by a randomized double-blind placebo-controlled trial. ${ }^{31)}$ In the present study, statistically significant reductions in serum LDL-C and non-HDL-C levels by ramelteon $(8 \mathrm{mg} / \mathrm{d})$ for 8 weeks or longer have been shown in general patients with insomnia including DM (Fig. 2), thus providing further evidence of ramelteon to improve serum lipid profiles even in patient population including DM. As a control, furthermore, we have similarly investigated serum lipid profiles after treatment with other sleep-promoting drugs. Since other sleep-promoting drugs did not significantly alter serum LDL-C or non-HDL-C levels (Fig. 2), the observed improvement appeared to be specific for ramelteon, but not resulting from general improvement of sleep status. To the best of our knowledge, this is the first report which showed the statistically significant lowering of serum LDL-C and non-HDL-C levels by ramelteon, comparing the effects of other sleep-promoting drugs. Furthermore, longer treatment with ramelteon, such as for 9-24 weeks, showed more profound LDL-C and non-HDL-C reductions (Figs. 3, 4). Serum HDL-C levels, in contrast, were not significantly altered by ramelteon; however, other sleep-promoting drugs showed early and transient decreases in serum HDL-C levels (Fig. 5), suggesting that treatment with other sleep-promoting drugs than ramelteon may cause transient increases in the CHD and ASCVD risk. As additional controls, we have examined changes in serum lipid profiles 2-24 weeks before the treatment with ramelteon or other sleep-promoting drugs, and did not find any significant changes in the serum lipid profiles before the initiation of the drug treatment. Thus, the observed changes in the serum lipid profiles appear to be independent of other factors, but depend upon ramelteon or other sleep-promoting drugs.

The patient characteristics of this study population (Tables $4,5)$ clearly indicated the high prevalence of DM. Since DM develops high serum TG levels accompanied by increases in small dense LDL particles and VLDL remnants resulting in elevated non-HDL-C levels, significant lowering in non-HDL$\mathrm{C}$, in addition to LDL-C, by ramelteon may be important to reduce $\mathrm{CHD}$ and $\mathrm{ASCVD}$ risk in patients with insomnia who are prevalently complicated with DM. In this study population, baseline mean serum LDL-C and non-HDL-C levels were $103.1-109.2$ and $138.8-133.2 \mathrm{mg} / \mathrm{dL}$, respectively. According to the current guidelines in Japan, these target levels are below 120 and $150 \mathrm{mg} / \mathrm{dL}$, respectively, in patients with $\mathrm{DM}$, and below 100 and $130 \mathrm{mg} / \mathrm{dL}$, respectively, in patients with CHD. ${ }^{21)}$ Almost 60 and $30 \%$ of this study population, as a whole, had DM and CHD, respectively; significant portions of the patients were supposed to reach neither their target serum LDL-C nor non-HDL-C levels, although almost a half of the patients were treated with lipid-lowering medication. Thus, additional serum lipid improvement by ramelteon is considered to be clinically significant. Reduced incidences of CHD or ASCVD by ramelteon should be explored by largescale clinical studies in the future.

Because patients with regular dialysis for chronic renal failure, malignant diseases treated with cytotoxic anti-cancer drugs or under seriously ill conditions do not necessary require strict serum LDL-C or non-HDL-C management, and their serum lipoprotein profiles may be influenced by the natural courses of their diseases, these patients have been excluded from the present study.

In addition, ramelteon did not show any HDL-C lowering effects, although significant and transient reduction in the HDL-C level was observed in the other sleep-promoting drug group (Figs. 2, 5). These results may provide a novel therapeutic recommendation for patients with insomnia accompanied by DM or at high risks for CHD and ASCVD. The precise mechanisms for this early and transient HDL-C reduction remain unknown; however, physical inactivity caused by such sleep-promoting drugs might have affected the serum HDL-C reduction. In any case, these results show the more beneficial effects of ramelteon on serum lipid profiles than other sleep-promoting drugs, in these high-risk patients. Since this is a retrospective study, limited numbers of patients were enrolled because of the exclusion criteria in order to eliminate factors which can affect serum lipid levels. Thus, limitation of this study may include the fact that very limited numbers of patients were enrolled. Therefore, large-scale prospective studies would also be needed to conclude the beneficial effects of ramelteon on lipoprotein metabolism, as well as CHD and ASCVD risk reduction, in humans.

A previous animal study with a hypercholesterolemic rat model has indicated that intraperitoneal injection of melatonin reduced serum LDL-C by enhancing cholesterol clearance but not suppressing cholesterol biosynthesis. ${ }^{36)}$ On the other hand, another study showed the possibility that melatonin inhibited absorption of cholesterol. ${ }^{37)}$ MT1 and MT2 receptors have been shown to be expressed in adipocytes, pancreatic islet cells and coronary arteries, in addition to brain. ${ }^{38-41)}$ However, precise molecular mechanisms involved in ramelteon-induced serum LDL-C and non-HDL-C reduction remain to be explored. Further studies are necessary to elucidate this point by suitable experimental animal models.

\section{CONCLUSION}

In conclusion, the present study demonstrates that ramelteon specifically reduces serum LDL-C and non-HDL-C levels, and thus may contribute to the CHD and ASCVD risk reduction in patients with insomnia.

Conflict of Interest The authors declare no conflict of interest.

\section{REFERENCES}

1) Ohayon MM. Epidemiology of insomnia: what we know and what we still need to learn. Sleep Med. Rev., 6, 97-111 (2002).

2) Ford ES, Cunningham TJ, Giles WH, Croft JB. Trends in insomnia and excessive daytime sleepiness among U.S. adults from 2002 to 
2012. Sleep Med., 16, 372-378 (2015).

3) Pallesen S, Sivertsen B, Nordhus IH, Bjorvatn B. A 10-year trend of insomnia prevalence in the adult Norwegian population. Sleep Med., 15, 173-179 (2014).

4) Calem M, Bisla J, Begum A, Dewey M, Bebbington PE, Brugha T, Cooper C, Jenkins R, Lindesay J, McManus S, Meltzer H, Spiers N, Weich S, Stewart R. Increased prevalence of insomnia and changes in hypnotics use in England over 15 years: analysis of the 1993, 2000, and 2007 National Psychiatric Morbidity Surveys. Sleep, 35, 377-384 (2012).

5) Liu X, Uchiyama M, Kim K, Okawa M, Shibui K, Kudo Y, Doi Y, Minowa M, Ogihara R. Sleep loss and daytime sleepiness in the general adult population of Japan. Psychiatry Res., 93, 1-11 (2000).

6) Kaneita Y, Uchiyama M, Yoshiike N, Ohida T. Associations of usual sleep duration with serum lipid and lipoprotein levels. Sleep, 31, 645-652 (2008).

7) Toyama Y, Chin K, Chihara Y, Takegami M, Takahashi K-I, Sumi K, Nakamura T, Nakayama-Ashida Y, Minami I, Horita S, Oka Y, Wakamura T, Fukuhara S-I, Mishima M, Kadotani H. Association between sleep apnea, sleep duration, and serum lipid profile in an urban, male, working population in Japan. Chest, 143, 720-728 (2013).

8) Sofi F, Cesari F, Casini A, Macchi C, Abbate R, Gensini GF. Insomnia and risk of cardiovascular disease: a meta-analysis. Eur. J. Prev. Cardiol., 21, 57-64 (2014).

9) Ministry of Health, Labour and Welfare; Tokyo, Japan: 2018 "Annual health, labour and welfare report 2017. Overview of the system and the basic statistics.": 〈https://www.mhlw.go.jp/english/wp/wp-hw11/dl/01e.pdf), cited September 5, 2018.

10) WHO (World Health Organization); Geneva, Switzerland. 2018 "The top 10 causes of death.": 〈http://www.who.int/mediacentre/ factsheets/fs310/en/s, cited September 5, 2018

11) Kannel WB, Dawber TR, Kagan A, Revotskie N, Stokes J 3rd. Factors of risk in the development of coronary heart disease-six year follow-up experience. The Framingham Study. Ann. Intern. Med., 55, 33-50 (1961).

12) Imano H, Noda H, Kitamura A, Sato S, Kiyama M, Sankai T, Ohira T, Nakamura M, Yamagishi K, Ikeda A, Shimamoto T, Iso H. Lowdensity lipoprotein cholesterol and risk of coronary heart disease among Japanese men and women: the Circulatory Risk in Communities Study (CIRCS). Prev. Med., 52, 381-386 (2011).

13) Miller M, Ginsberg HN, Schaefer EJ. Relative atherogenicity and predictive value of non-High-density lipoprotein cholesterol for coronary heart disease. Am. J. Cardiol., 101, 1003-1008 (2008).

14) Imamura $T$, Doi $Y$, Ninomiya $T$, Hata J, Nagata M, Ikeda F, Mukai N, Hirakawa Y, Yoshida D, Fukuhara M, Kitazono T, Kiyohara Y. Non-high-density lipoprotein cholesterol and the development of coronary heart disease and stroke subtypes in a general Japanese population: the Hisayama Study. Atherosclerosis, 233, 343-348 (2014).

15) Grundy SM, Cleeman JI, Merz CNB, Brewer HB Jr, Clark LT, Hunninghake DB, Pasternak RC, Smith SC Jr, Stone NJ; National Heart, Lung, and Blood Institute, American College of Cardiology Foundation, American Heart Association; American College of Cardiology Foundation; American Heart Association. Implications of recent clinical trials for the National Cholesterol Education Program Adult Treatment Panel III guidelines. Circulation, 110, 227-239 (2004).

16) Li C, Ford ES, Tsai J, Zhao G, Balluz LS, Gidding SS. Serum Nonhigh-density lipoprotein cholesterol concentration and risk of death from cardiovascular diseases among U.S. adults with diagnosed diabetes: The Third National Health and Nutrition Examination Survey linked mortality study. Cardiovasc. Diabetol., 10, 46 (2011).

17) The Lipid Research Clinics Coronary Primary Prevention Trial results. II. The relationship of reduction in incidence of coronary heart disease to cholesterol lowering. JAMA, 251, 365-374 (1984).
18) Scandinavian Simvastatin Survival Study Group. Randomised trial of cholesterol lowering in 4444 patients with coronary heart disease: the Scandinavian Simvastatin Survival Study (4S). Lancet, 344, 1383-1389 (1994).

19) Shepherd J, Cobbe SM, Ford I, Isles CG, Lorimer AR, MacFarlane PW, McKillop JH, Packard CJ; West of Scotland Coronary Prevention Study Group. Prevention of coronary heart disease with pravastatin in men with hypercholesterolemia. West of Scotland Coronary Prevention Study Group. N. Engl. J. Med., 333, 1301-1307 (1995).

20) Cannon CP, Blazing MA, Giugliano RP, McCagg A, White JA, Theroux P, Darius H, Lewis BS, Ophuis TO, Jukema JW, De Ferrari GM, Ruzyllo W, De Lucca P, Im K, Bohula EA, Reist C, Wiviott SD, Tershakovec AM, Musliner TA, Braunwald E, Califf RM; IMPROVE-IT Investigators. Ezetimibe added to statin therapy after acute coronary syndromes. N. Engl. J. Med., 372, 2387-2397 (2015). 21) Teramoto T, Sasaki J, Ishibashi S, Birou S, Daida H, Dohi S, Egusa G, Hiro T, Hirobe K, Iida M, Kihara S, Kinoshita M, Maruyama C, Ohta T, Okamura T, Yamashita S, Yokode M, Yokote K, Japan Atherosclerosis Society. Executive summary of the Japan Atherosclerosis Society (JAS) guidelines for the diagnosis and prevention of atherosclerotic cardiovascular diseases in Japan-2012 version. $J$. Atheroscler. Thromb., 20, 517-523 (2013).

22) Reiner Z, Catapano AL, De Backer G, Graham I, Taskinen MR, Wiklund O, Agewall S, Alegria E, Chapman MJ, Durrington P, Erdine S, Halcox J, Hobbs R, Kjekshus J, Filardi PP, Riccardi G, Storey RF, Wood D, Bax J, Vahanian A, Auricchio A, Baumgartner H, Ceconi C, Dean V, Deaton C, Fagard R, Filippatos G, FunckBrentano C, Hasdai D, Hobbs R, Hoes A, Kearney P, Knuuti J, Kolh P, McDonagh T, Moulin C, Poldermans D, Popescu BA, Reiner Z, Sechtem U, Sirnes PA, Tendera M, Torbicki A, Vardas P, Widimsky $\mathrm{P}$, Windecker S, Reviewers D, Funck-Brentano C, Poldermans D, Berkenboom G, De Graaf J, Descamps O, Gotcheva N, Griffith K, Guida GF, Gulec S, Henkin Y, Huber K, Kesaniemi YA, Lekakis J, Manolis AJ, Marques-Vidal P, Masana L, McMurray J, Mendes M, Pagava Z, Pedersen T, Prescott E, Rato Q, Rosano G, Sans S, Stalenhoef A, Tokgozoglu L, Viigimaa M, Wittekoek ME, Zamorano JL; European Association for Cardiovascular Prevention \& Rehabilitation; ESC Committee for Practice Guidelines (CPG) 2008-2010 and 2010-2012 Committees. ESC/EAS Guidelines for the management of dyslipidaemias: The Task Force for the management of dyslipidaemias of the European Society of Cardiology (ESC) and the European Atherosclerosis Society (EAS). Eur. Heart J., 32, 1769-1818 (2011)

23) Nakazaki C, Noda A, Koike Y, Yamada S, Murohara T, Ozaki N. Association of Insomnia and Short Sleep Duration With Atherosclerosis Risk in the Elderly. Am. J. Hypertens., 25, 1149-1155 (2012).

24) Ayas NT, White DP, Manson JE, Stampfer MJ, Speizer FE, Malhotra A, Hu FB. A prospective study of sleep duration and coronary heart disease in women. Arch. Intern. Med., 163, 205-209 (2003).

25) Brzezinski A. Melatonin in humans. N. Engl. J. Med., 336, 186-195 (1997).

26) Miyamoto M. Pharmacology of ramelteon, a selective MT1/MT2 receptor agonist: a novel therapeutic drug for sleep disorders. CNS Neurosci. Ther., 15, 32-51 (2009).

27) Cajochen C. TAK-375 Takeda. Curr. Opin. Investig. Drugs. 2000., 6, 114-121 (2005).

28) Koziróg M, Poliwczak AR, Duchnowicz P, Koter-Michalak M, Sikora J, Broncel M. Melatonin treatment improves blood pressure, lipid profile, and parameters of oxidative stress in patients with metabolic syndrome. J. Pineal Res., 50, 261-266 (2011).

29) Nduhirabandi F, du Toit EF, Lochner A. Melatonin and the metabolic syndrome: a tool for effective therapy in obesity-associated abnormalities? Acta Physiol., 205, 209-223 (2012).

30) Goyal A, Terry PD, Superak HM, Nell-Dybdahl CL, Chowdhury R, Phillips LS, Kutner MH. Melatonin supplementation to treat the metabolic syndrome: a randomized controlled trial. Diabetol. 
Metab. Syndr., 6, 124 (2014).

31) Borba CPC, Fan X, Copeland PM, Paiva A, Freudenreich O, Henderson DC. Placebo-controlled pilot study of ramelteon for adiposity and lipids in patients with schizophrenia. J. Clin. Psychopharmacol., 31, 653-658 (2011).

32) Lewy AJ, Wehr TA, Goodwin FK, Newsome DA, Markey SP. Light suppresses melatonin secretion in humans. Science, 210, 1267-1269 (1980).

33) Uzun S, Kozumplik O, Jakovljević M, Sedić B. Side effects of treatment with benzodiazepines. Psychiatr. Danub., 22, 90-93 (2010).

34) Weaver MF. Prescription Sedative Misuse and Abuse. Yale J. Biol. Med., 88, 247-256 (2015).

35) Kang DY, Park S, Rhee CW, Kim YJ, Choi NK, Lee J, Park BJ. Zolpidem use and risk of fracture in elderly insomnia patients. $J$. Prev. Med. Pub. Health, 45, 219-226 (2012).

36) Chan TY, Tang PL. Effect of melatonin on the maintenance of cholesterol homeostasis in the rat. Endocr. Res., 21, 681-696 (1995).
37) Hussain SA-R. Effect of melatonin on cholesterol absorption in rats. J. Pineal Res., 42, 267-271 (2007).

38) Brydon L, Petit L, Delagrange P, Strosberg AD, Jockers R. Functional expression of MT2 (Mellb) melatonin receptors in human PAZ6 adipocytes. Endocrinology, 142, 4264-4271 (2001).

39) Ramracheya RD, Muller DS, Squires PE, Brereton H, Sugden D, Huang GC, Amiel SA, Jones PM, Persaud SJ. Function and expression of melatonin receptors on human pancreatic islets. J. Pineal Res., 44, 273-279 (2008).

40) Ekmekcioglu C, Thalhammer T, Humpeler S, Mehrabi MR, Glogar HD, Hölzenbein T, Markovic O, Leibetseder VJ, Strauss-Blasche G, Marktl W. The melatonin receptor subtype MT2 is present in the human cardiovascular system. J. Pineal Res., 35, 40-44 (2003).

41) Mazzucchelli C, Pannacci M, Nonno R, Lucini V, Fraschini F, Stankov BM. The melatonin receptor in the human brain: cloning experiments and distribution studies. Brain Res. Mol. Brain Res., 39, 117-126 (1996). 